\title{
Green Tea
}

National Cancer Institute

\section{Source}

National Cancer Institute. Green Tea. NCI Thesaurus. Code C67048.

Tea derived from the dried leaves of the plant Camellia sinensis with potential antioxidant, chemopreventive, and lipid-lowering activities. Green tea contains polyphenols that are believed to be responsible for its chemopreventive effect. The polyphenol fraction contains mainly Epigallocatechin-3-gallate (EGCG) and other catechins, such as epicatechin (EC), gallocatechin gallate (GCG), epigallocatechin (EGC), and epicatechin gallate (ECG). Green tea polyphenols act as antioxidants and free radical scavengers and may affect enzymes involved in cellular replication and tumor angiogenesis by modulating angiogenic factors, such as vascular endothelial g rowth factor (VEGF). 